\title{
Melhoria do desempenho da Fatoração Controlada de Cholesky no precondicionamento de Sistemas Lineares oriundos dos Métodos de Pontos Interiores
}

\author{
Lino Marcos da Silva, \\ Colegiado de Engenharia Elétrica, CENEL, UNIVASF, \\ 48902-300, Juazeiro, BA \\ E-mail: lino.silva@univasf.edu.br, \\ Aurelio Ribeiro Leite de Oliveira \\ Depertamento de Matemática Aplicada, IMECC, UNICAMP \\ 13083-859, Campinas, SP \\ E-mail: aurelio@ime.unicamp.br.
}

\begin{abstract}
Resumo: O método de pontos interiores para programação linear resolve em poucas iterações problemas de grande porte. No entanto, requer a cada iteração a resolução de dois sistemas lineares, os quais possuem a mesma matriz de coeficientes. Essa etapa se constitui no passo mais caro do método por aumentar consideravelmente o tempo de processamento e a necessidade de armazenamento de dados. Reduzir o tempo de solução dos sistemas lineares é, portanto, uma forma de melhorar o desempenho do método. De um modo geral, problemas de programação linear de grande porte possuem matrizes esparsas, e os sistemas lineares a serem resolvidos são simétricos positivos definidos. Assim métodos iterativos como o método dos gradientes conjugados precondicionado podem ser utilizados na resolução dos mesmos. Além disso, fatores de Cholesky incompletos podem ser utilizados como precondicionadores do problema. Por outro lado fatoraçôes incompletas podem sofrer falhas na diagonal durante o processo de fatoração. Quando tais falhas ocorrem, é necessário efetuar uma correção, o que em geral ocasiona um aumento no tempo de precondicionamento quer seja devido a reconstrução do precondicionador, quer seja devido a perda de qualidade do mesmo. Neste trabalho propomos uma nova abordagem para a correção de falhas na diagonal ocorridas durante a fatoração controlada de Cholesky. Resultados computacionais mostraram que a técnica pode reduzir significativamente o tempo de resolução de problemas de programação linear via método de pontos interiores.
\end{abstract}

Palavras-chave: Programação Linear, Método de Pontos Interiores, Precondicionadores

\section{Introdução}

Considere o sistema linear $\mathbf{A} x=r$ com a matriz de coeficientes $\mathbf{A}$ sendo simétrica positiva definida. Precondicionar esse sistema linear consiste em determinar uma matriz $N$ adequada tal que o novo sistema $N^{-1} \mathbf{A} N^{-T} \tilde{x}=\tilde{r}$, onde $\tilde{x}=N^{T} x$ e $\tilde{r}=N^{-1} r$, é mais fácil de resolver. A matriz $N$ é chamada de precondicionador e funciona tanto melhor, quanto mais próximos da unidade estiverem os autovalores da matriz $N^{-1} \mathbf{A} N^{-T}$ ou pelo menos agrupados em poucos clusters longes da origem. A fatoração incompleta é umas das técnicas de precondicionamento muito utilizada no precondicionamento de sistemas lineares pelo fato de funcionarem bem para uma grande variedade de problemas e também pela simplicidade com que os mesmos podem ser construídos.

Seja $A$ uma matriz simétrica positiva definida, uma decomposição triangular de $A$ é dada por $A=L D U^{T}$ sendo $D=\operatorname{diag}\left(d_{1}, \ldots, d_{n}\right), d_{i}>0$ e os fatores $L$ e $U$ triangulares inferior e superior, respectivamente, ambos com diagonal unitária. A fatoração incompleta de $A$ é um método pelo 
qual obtemos uma matriz $M$ tal que $M=\tilde{L} \Delta \tilde{U}^{T}$ e $A=M+R$, onde $R$ é uma matriz resto, $\Delta=\operatorname{diag}\left(\delta_{1}, \ldots, \delta_{n}\right)$ e $\tilde{L}$ e $\tilde{U}$ são fatores triangulares inferior e superior, respectivamente, ambos com diagonal unitária. Se $A$ é simétrica, então fazendo $\hat{L}=\tilde{L} \Delta^{1 / 2}$ obtem-se $M=\hat{L} \hat{L}^{T}$, a qual é denominada de Fatoração de Cholesky Incompleta. Para uma matriz $A$ simétrica e positiva definida existe a fatoração de Cholesky $A=L L^{T}$, onde $L$ é uma matriz triangular inferior com todos os elementos da diagonal positivos. No entanto, quando se trata de fatorações incompletas, pode não existir um fator $\hat{L}$ tal que $A=\hat{L} \hat{L}^{T}+R$, pois estas são suscetíveis à falhas na diagonal. Tais falhas são provocadas pela presença de pivôs não positivos ou muito pequenos em fatorações incompletas, e é reconhecidamente um problema sério [1]. Algumas alternativas tem sido propostas para contornar o problema. Neste trabalho veremos duas abordagens adotadas para evitar falhas na diagonal durante fatorações incompletas, apresentaremos uma nova proposta de correção na diagonal e a aplicamos no precondicionamento de sistemas lineares oriundos de métodos de pontos interiores. A correção proposta será aplicada a Fatoração Controlada de Cholesky, a FCC, a qual é um precondicionador proposto por Campos e Birkett [3] como uma variação da fatoração de Cholesky incompleta. Na FCC o número de elementos nas colunas de $\tilde{L}$ pode ser controlado por um parâmetro $\eta$, de modo que, $\tilde{L}$ poderá ter mais ou menos elementos que $A$. Esse parâmetro pode ser dado em função da quantidade de espaço disponível. Se a demanda por memória não for crítica, pode ser construído um precondicionador com mais elementos que $A$, acelerando assim a convergência do método iterativo.

\section{Correção na diagonal}

Aumentar a dominância diagonal de $A$, acrescentando um incremento aos elementos da diagonal da matriz, é uma das principais técnicas utilizadas para efetuar uma correção na diagonal durante fatorações incompletas. Essa estratégia pode ser local, quando um valor positivo é somado apenas na posição da diagonal de $A$ onde ocorreu a falha; ou global, quando todos os elementos da diagonal recebem um acréscimo. Um exemplo simples dessa técnica é trocar um pivô pequeno ou negativo em cada etapa $i$ da fatoração, onde o mesmo aparece, por algum valor $p_{i}>0$. Esta estratégia local é motivada pela esperança de que se apenas poucos pivôs são instáveis a fatoração resultante ainda pode render um precondicionador satisfatório. Apesar da simplicidade da técnica em si mesma, a escolha de tal $p_{i}$ não é fácil pois um valor de $p_{i}$ muito grande, implica em $\left\|A-\hat{L} \hat{L}^{T}\right\|_{F}$ muito grande e isso resulta na imprecisão do precondicionador. Por outro lado, a escolha de um $p_{i}$ muito pequeno pode gerar instabilidade na resolução dos sistemas triangulares com $\hat{L}$ e $\hat{L}^{T}$. Um exemplo mais elaborado dessa técnica é efetuar um incremento global em $A$ antes de tentar uma nova fatoração. Assim a nova fatoração incompleta é calculada com a matriz $\hat{A}=A+\alpha I, \alpha>0$ e $I$ a matriz identidade de ordem $n$. Se para um determinado $\alpha$, a fatoração falha, então o mesmo é aumentado de algum valor e o processo é repetido até que a fatoração incompleta seja calculada com sucesso. Apesar de não ser fácil a escolha de um $\alpha$ adequado, é possível, afirmar que existe um $\alpha^{*}>0$ para o qual a fatoração da matriz $\hat{A}=A+\alpha^{*} I$ existe. Por exemplo, $\alpha^{*}$ pode ser o menor $\alpha$ para o qual a matriz $\hat{A}$ seja diagonalmente dominante. Mas como dominância diagonal é uma condição suficiente, mas não necessária para a existência de fatorações incompletas, é possível determinar um $\alpha$ tal que $0<\alpha<\alpha^{*}$ e para o qual a fatoração incompleta seja realizada com sucesso. Em geral $\alpha$ é escolhido por meio de tentativa e erro, prática que torna o método caro e configura a sua escolha como sendo a principal desvantagem dessa técnica. A seguir apresentamos as duas principais abordagens para a escolha do $\alpha$.

\subsection{A escolha do $\alpha$ por incremento linear}

O algoritmo com essa abordagem soma um valor fixo $\lambda$ à diagonal da matriz e repete o processo até a fatoração incompleta ser realizada com sucesso. Dessa forma, o parâmetro pode ser definido pela fórmula $\alpha_{i}=\lambda i$ onde $\lambda$ é um número positivo fixo e $i=1, \ldots, T_{\max }$. O número $T_{\max }$ indica o número máximo permitido de reinicio da fatoração. 


\subsection{A escolha do $\alpha$ por incremento exponencial}

Quando um elemento da diagonal é muito pequeno ou não positivo, essa abordagem soma aos elementos da diagonal de $A$, o valor dado por $\alpha_{i}=\lambda 2^{i}$ e recomeça a fatoração. Neste caso $\lambda$ é uma constante positiva e $i=1, \ldots, T_{\max }$. Desse modo espera-se que o algoritmo encontre mais rapidamente um valor adequado de $\alpha$ do que a abordagem linear.

A principal desvantagem das abordagens anteriores é a possibilidade do incremento na diagonal ser realizado várias vezes, com reinicio da fatoração, até que a mesma seja concluída com sucesso ou até que o número de reinício máximo permitido $T_{\max }$ seja atingido. De um modo geral, começam-se as correções com um valor de $\alpha$ pequeno e reinicia-se a fatoração. Se a fatoração falha novamente, aumenta-se o valor de $\alpha$ e reinicia-se outra vez a fatoração. O processo continua e o valor de $\alpha$ é aumentado progressivamente até atingir um valor $\bar{\alpha}$ para o qual a fatoração incompleta exista ou uma certa tolerância seja atingida.

\subsection{Uma nova abordagem para a escolha do $\alpha$}

A nossa proposta pretende combinar dois importantes aspectos: efetuar correções com valores pequenos de $\alpha$ e reduzir o número de reinícios necessário da fatoração até que a mesma seja completada com sucesso. Para tanto propomos uma escolha de $\alpha$ do seguinte modo: suponha que durante o processo de fatoração $A=L D L^{T}$, ocorra uma falha na diagonal na coluna $j$ da matriz $A$, isto é, $d_{j j} \leq 0$. Vamos começar com um valor pequeno de $\alpha$, digamos $\alpha_{i}$, e aumentá-lo progressivamente, usando a abordagem exponencial, até encontrar um $\bar{\alpha}$ tal que $d_{j j}+\bar{\alpha}>0$. Provavelmente tal valor de $\alpha$ adicionado a diagonal de $A$ irá permitir a continuação do processo de fatoração além da coluna $j$, e possivelmente possibilitar a conclusão da fatoração. No entanto, é possível que exista valores de $\alpha$ menores do que $\bar{\alpha}$ tais que a fatoração de $A+\alpha I$ existe e produziriam melhores precondicionadores do que $\bar{\alpha}$, pois a correção na diagonal seria menor. Sendo assim, para atenuar esse problema usaremos uma estratégia de backtracking e propomos a seguinte fórmula para escolha de $\alpha$ :

$$
\alpha=\tau \alpha_{i}
$$

onde $\tau \in(0,1], i=1, \ldots, T_{\max }$ e $\alpha_{i}=\lambda 2^{i}$. Assim, se durante a fatoração, ocorre uma falha na coluna $j$, calculamos um $\alpha_{i}$ tal que $d_{j j}+\alpha_{i}>0$ e reduzimos o tamanho deste $\alpha_{i}$ multiplicando-o pelo fator $\tau$. A escolha do fator $\tau$ deve levar em consideração os dois aspectos mencionados anteriormente: deve permitir uma redução razoável do valor $\alpha_{i}$ para que tenhamos um bom precondicionador, mas essa redução não pode ser muito grande a ponto de que novas correções precisem ser efetuadas na mesma iteração. Esta proposta modifica a abordagem exponencial de duas formas: primeiro a abordagem refina a escolha do parâmetro $\alpha$ permitindo que a correção na diagonal possa ser realizada com um valor menor. Isso é importante porque mantém a qualidade do precondicionador; segundo, o número de refatorações pode ser reduzido de acordo com a escolha de $\tau$. Por exemplo, a escolha de $\tau=1$ impede o reinício da fatoração para a coluna em questão e isso gera ganho de tempo na construção do precondicionador.

\section{Aplicação no precondicionamento em métodos de pontos inte- riores}

O método de pontos interiores resolve o problema de programação linear

$$
\begin{array}{ll}
\min & c^{T} x \\
\text { s.a } & A x=b \\
& x \geq 0
\end{array}
$$

onde $A \in \mathbb{R}^{m x n}, x \in \mathbb{R}^{n}, c \in \mathbb{R}^{n}, b \in \mathbb{R}^{m}$ e a matriz $A$ com posto completo. Dizemos que o problema (1) está na forma padrão, o qual é também chamado de problema primal. Associado a este problema existe o problema dual que é dado por 


$$
\begin{array}{ll}
\max & b^{T} y \\
\text { s.a } & A^{T} y+z=c \\
& z
\end{array}
$$

O método preditor-corretor é uma das mais bem sucedidas variações de métodos de pontos interiores. Neste método as direções de busca são obtidas resolvendo dois sistemas lineares. Primeiro as direções afim-escala são calculadas

$$
\left[\begin{array}{ccc}
0 & I & A^{T} \\
Z & X & 0 \\
A & 0 & 0
\end{array}\right]\left[\begin{array}{c}
\Delta \tilde{x} \\
\Delta \tilde{z} \\
\Delta \tilde{y}
\end{array}\right]=\left[\begin{array}{c}
r_{d} \\
r_{a} \\
r_{p}
\end{array}\right] .
$$

onde $X=\operatorname{diag}(x), Z=\operatorname{diag}(z)$ e os resíduos são dados por $r_{p}=b-A x, r_{d}=c-A^{T} y-z$ e $r_{a}=-X Z e$. Então as direções $(\Delta x, \Delta y, \Delta z)$ são calculadas resolvendo o sistema (3) com $r_{a}$ substituído por $r_{c}=\mu e-X Z e-\Delta \tilde{X} \Delta \tilde{Z} e$ onde $\mu$ é o parâmetro de centragem e $e=(1,1, \ldots, 1)^{T}$.

O passo chave de uma iteração do método de pontos interiores é a solução de um sistema linear como (3) pois o mesmo absorve o maior custo computacional do método. No entanto, como os dois sistemas compartilham a mesma matriz dos coeficientes, podemos restringir as nossas discussões a apenas um dos sistemas lineares. Eliminado a variável $\Delta z$ o sistema fica reduzido a:

$$
\left[\begin{array}{cc}
-\Theta & A^{T} \\
A & 0
\end{array}\right]\left[\begin{array}{c}
\Delta x \\
\Delta y
\end{array}\right]=\left[\begin{array}{c}
r_{d}-X^{-1} r_{a} \\
r_{p}
\end{array}\right]
$$

onde $\Theta=X^{-1} Z$ é uma matriz diagonal de ordem $n$ e a matriz bloco-diagonal inferior 0 é de ordem $m$. Este sistema é chamado de sistema aumentado. Agora eliminando $\Delta x$ de (4) obtemos

$$
A \Theta^{-1} A^{T} \Delta y=r_{p}+A\left(\Theta^{-1} r_{d}-Z^{-1} r_{a}\right)
$$

que é chamado de sistema de equações normais.

\subsection{Abordagem para resolver os sistemas lineares}

A fatoração de Cholesky do sistema de equações normais é a abordagem mais utilizada nas implementações dos método de pontos interiores para o cálculo das direções de buscas. Contudo, o fator de Cholesky resultante pode ser muito menos esparso que a matriz original. Esse efeito, chamado fill-in, é indesejável principalmente quando se trata da resolução de problemas de grande porte, nos quais a questão do armazenamento de dados é crítica. Uma alternativa a fatoração de Cholesky é o uso do método dos gradientes conjugados. Este método, no entanto, é sensível ao condicionamento da matriz do sistema. Isto é, o mesmo não funciona bem quando aplicado em matrizes mal condicionadas. Por outro lado, o sistema linear (5) fica muito mal condicionado ao longo das iterações do método de pontos interiores, e desse modo, o método dos gradientes conjugados pode necessitar de muitas iterações ou até mesmo não convergir. Assim para que o método possa ser aplicado com sucesso, o sistema linear mal condicionado necessita ser precondicionado.

\subsubsection{Precondicionamento em método de pontos interiores}

Uma técnica que tem mostrado bons resultados no precondicionamento de método de pontos interiores é a proposta de precondicionamento híbrido de Bocanegra, Campos e Oliveira [2]. Esta abordagem reúne dois precondicionadores distintos que atuam em iterações distintas do método de pontos interiores. Primeiro, usa-se a FCC, que trabalha bem nas iterações iniciais do método de pontos interiores; e em seguida usa o precondicionador separador proposto por Oliveira e Sorensen [4], que trabalha bem nas iterações finais do método. Um momento crucial nessa abordagem é o da troca entre os precondicionadores. Uma heurística para efetuar essa 
troca foi desenvolvida por Velazco, Oliveira e Campos [5]. O método começa a trabalhar com o precondicionador FCC, e quando este já não tem um bom desempenho, por exemplo o número de iterações internas do gradientes conjugados ultrapassar um quarto do valor da dimensão do problema, ocorre a troca para o precondicionador separador.

\section{Resultados Computacionais}

Os resultados computacionais seguintes comparam o desempenho da abordagem de correção na diagonal proposta para a FCC, com a implementação original da mesma. Para os testes foi utilizado o código PCx, que é uma implementação de métodos de pontos interiores do tipo preditor-corretor para o problema de programa linear; e no qual está inserida a rotina que calcula a FCC, implementada em fortran 77. A versão utilizada incorpora a abordagem de precondicionamento híbrido proposta em [2]. Os problemas testes utilizados nesta abordagem de precondicionamento híbrida são provenientes das coleções NETLIB, QAPLIB e MISC. Além dessas informações, a Tabela 1 resume outros dados dos problemas como o número de linhas e de colunas, todos referentes aos problemas pré-processados. Os testes realizados com os problemas PDS foram realizados em um computador com processador Intel Corel i7-2600 CPU@3.40GHzx8 e 3,8GiB de memória RAM. Os demais foram testados em uma máquina com processador Intel Core i5-2410M CPU@2.30GHz 2 e 4GB RAM de memória.

Tabela 1: Problemas Testes

\begin{tabular}{lrrl||lrrr}
\hline \hline Problema & Linhas & Colunas & Coleção & Problema & Linhas & Colunas & Coleção \\
\hline els19 & 4350 & 13186 & QAPLIB & pds-02 & 2953 & 7535 & MISC \\
chr22b & 5587 & 10417 & QAPLIB & pds-06 & 9881 & 28655 & MISC \\
chr25a & 8149 & 15325 & QAPLIB & pds-10 & 16558 & 48763 & MISC \\
scr15 & 2234 & 6210 & QAPLIB & pds-20 & 32276 & 106180 & MISC \\
scr20 & 5079 & 15980 & QAPLIB & pds-40 & 64265 & 214385 & MISC \\
rou20 & 7359 & 37640 & QAPLIB & pds-60 & 96503 & 332862 & MISC \\
agg2 & 514 & 750 & NETLIB & pds-80 & 126109 & 430800 & MISC \\
agg3 & 514 & 750 & NETLIB & pds-100 & 156243 & 514577 & MISC \\
bn12 & 1964 & 4008 & NETLIB & & & & \\
\hline
\end{tabular}

Para os problemas QAPLIB, o número de correções que acontecem na diagonal pode ser muito alto. Como apresentado na Tabela 2, o problema rou20 por exemplo, tem a fatoração incompleta reiniciada quinze vezes durante a sétima iteração do método de pontos interiores. Além disso, a fatoração precisa ser recomeçada pelo menos trezes vezes em cada uma das próximas cinco iterações, contabilizando um total de setenta e um reinício da FCC.

Tabela 2: Número de correções na diagonal e reinícios da fatoração.

\begin{tabular}{ccccccccccccccccccc}
\hline \hline Iteração & 0 & 1 & 2 & 3 & 4 & 5 & 6 & 7 & 8 & 9 & 10 & 11 & 12 & 13 & 14 & 15 & 16 & 17 \\
\hline els19 & & & & & & & & & 14 & 14 & 13 & 13 & 12 & 12 & & & \\
ch25a & & & & & & & & & & & & 13 & 13 & 11 & 11 & 11 & & \\
scr15 & & & & & & & & 13 & 13 & 12 & 12 & 12 & & & & & & \\
rou20 & & & & & & & 15 & 14 & 13 & 13 & 13 & 13 & & & & & \\
\hline
\end{tabular}

Os problemas pds são exemplos de problemas para os quais o critério de troca de fases, na abordagem híbrida, não é atingido. Isto acontece porque a FCC apresenta bom desempenho durante todas as iterações de pontos interiores. Desse modo, esses problemas se adequam bem ao estudo do desempenho da correção na diagonal ora proposta. Os resultados computacionais apresentados comparam o desempenho da abordagem híbrida proposta em [2], o qual denotaremos daqui em diante como FCC original, e uma versão modificada da mesma com a nova 
proposta de correção na diagonal e com os seguintes valores de $\tau: \tau=0,85, \tau=0,90, \tau=0,95$ e $\tau=1=\max$. Nas tabelas seguintes $I_{\tau}$ indica o número de iterações e $T_{\tau}$ tempo de resolução dos problemas para os respectivos valores de $\tau$.

Tabela 3: Problemas PDS

\begin{tabular}{lcccc||rrrr}
\hline PROBLEMA & $I_{F C C-\text { orig }}$ & $I_{0,85}$ & $I_{0,90}$ & $I_{\tau_{\max }}$ & $T_{F C C-\text { orig }}$ & $T_{0,85}$ & $T_{0,90}$ & $T_{\tau_{\max }}$ \\
\hline \hline pds-02 & 25 & 25 & 26 & 25 & 0,49 & 0,49 & 0,52 & 0,49 \\
pds-06 & 39 & 39 & 39 & 39 & 7,55 & $\mathbf{7 , 4 0}$ & 7,58 & 7,52 \\
pds-10 & 47 & 48 & 47 & 47 & 16,16 & 16,85 & 16,19 & $\mathbf{1 6 , 0 1}$ \\
pds-20 & 60 & 61 & 61 & 60 & 187,53 & $\mathbf{1 8 4 , 7 6}$ & 223,26 & 188,17 \\
pds-40 & 78 & 78 & 78 & 78 & 590,02 & $\mathbf{5 6 5 , 3 2}$ & 650,44 & 567,87 \\
pds-60 & 84 & 86 & 84 & 84 & 2082,23 & 2183,66 & 2034,64 & $\mathbf{2 0 0 6 , 8 9}$ \\
pds-80 & 83 & 83 & 83 & 83 & 2876,30 & 2756,50 & $\mathbf{2 7 7 2 , 8 4}$ & 2829,33 \\
pds-100 & 85 & 87 & 84 & 85 & 5173,30 & 5184,36 & $\mathbf{4 7 3 6 , 6 1}$ & 5142,80 \\
\hline
\end{tabular}

A Tabela 3 compara os resultados obtidos no precondicionamento dos problemas pds. Os resultados mostram que para os problemas de maior porte, como o pds-60, pds- 80 e pds- 100 , o tempo de resolução de cada problema é reduzido significativamente quando é utilizada a nova abordagem com os valores $\tau=0,90$ e $\tau=1=\max$. Isso indica que houve uma redução no tempo de preocondicionamento do mesmos.

Os resultados reportados na Tabela 4 indicam que a abordagem proposta para a correção na diagonal da FCC, também reduz o tempo de resolução quando as duas fases do precondicionamento híbrido são realizadas. Os resultados mostram que é possível obter uma redução no tempo do precondicionamento para quase todos os problemas quando adota-se a nova abordagem com algum valor para $\tau$. Além disso, a Tabela 4 mostra três problemas da coleção NETLIB que não são resolvidos quando a FCC original é utilizada, mas são resolvidos pela nova abordagem.

Tabela 4: Problemas QAP e NETlib

\begin{tabular}{lcccc||rrrr}
\hline PROBLEMA & $I_{\text {orig }}$ & $I_{0,85}$ & $I_{0,90}$ & $I_{\tau_{\max }}$ & $T_{\text {orig }}$ & $T_{0,85}$ & $T_{0,90}$ & $T_{\tau_{\max }}$ \\
\hline \hline els19 & 31 & 31 & 31 & 31 & 540,2 & $\mathbf{5 3 , 9 0}$ & 53,91 & 54,75 \\
chr22b & 29 & 29 & 29 & 29 & 23,52 & 20,68 & $\mathbf{2 0 , 1 2}$ & 22,08 \\
chr25a & 29 & 28 & 28 & 28 & 51,84 & $\mathbf{4 5 , 2 2}$ & 59,36 & 63,36 \\
scr15 & 24 & 24 & 24 & 24 & 9,33 & $\mathbf{8 , 0 2}$ & 11,37 & 8,56 \\
scr20 & 21 & 21 & 21 & 21 & 74,42 & $\mathbf{6 8 , 6 4}$ & 79,92 & 79,22 \\
rou20 & 24 & 24 & 24 & 24 & $\mathbf{9 5 8 , 9 3}$ & 1085,92 & 1052,93 & 1081,82 \\
agg2 & $\mathrm{F}$ & $\mathrm{F}$ & 30 & 31 & - & - & $\mathbf{0 , 3 2}$ & 0,38 \\
agg3 & $\mathrm{F}$ & 24 & 31 & $\mathrm{~F}$ & - & $\mathbf{0 , 3 5}$ & 0,43 & - \\
bn12 & $\mathrm{F}$ & 37 & 37 & 36 & - & 4,56 & 4,54 & $\mathbf{4 , 0 0}$ \\
\hline
\end{tabular}

A curva de desempenho da FCC original e da FCC modificada com $\tau=0,85, \tau=0,90$ e $\tau=1$, pode ser visualizada na Figura 1. Observa-se que a FCC apresenta um melhor desempenho para $\tau=0,85$ e que a FCC-original tem desempenho inferior ao das três versões modificadas para os problemas testados.

\section{Conclusões e trabalhos futuros}

Uma nova abordagem para a correção de falhas na diagonal durante a fatoração controlada de Cholesky foi proposta. A nova abordagem consiste em efetuar correções na diagonal de modo a evitar o reinício da fatoração, já que essa ação aumenta consideravelmente o custo computacional do fator incompleto de Cholesky. O fator calculado através da abordagem proposta foi aplicado no precondicionamento de sistemas lineares oriundos de métodos de pontos interiores. Resultados 


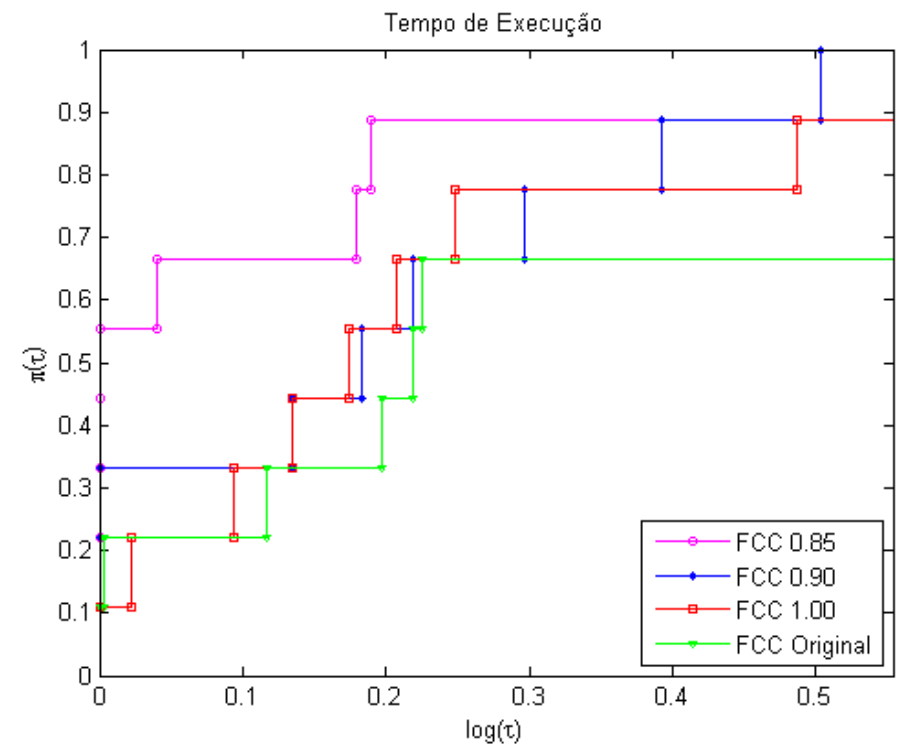

Figura 1: Performance profile: problemas QAP e NETLIB

obtidos com problemas de programação linear de grande porte indicam que é possível reduzir o tempo de resolução dos problemas com o uso da técnica proposta. Futuramente a técnica será combinada à fatoração controlada de Cholesky com o objetivo de obter precondicionadores eficientes através da atualizações de outros pré-existentes. Além disso, como fatorações incompletas são precondicionadores universais, a técnica também será testada no precondicionamento de sistemas lineares oriundos de outros problemas.

\section{Agradecimentos}

Os autores agradecem ao CNPq, à CAPES e à FAPESP o apoio recebido para o desenvolvimento deste trabalho.

\section{Referências}

[1] M. Benzi, G. H. Golub, and J. Liesen. Numerical solution of saddle point problems.Acta Numerica, 14:1-137, 52005.

[2] S. Bocanegra, F.F Campos, and A.R.L Oliveira. Using a hybrid preconditioner for solvinglarge-scale linear systems arising from interior point methods. Computational Optimization and Applications, 36:149-164, 2007.

[3] F.F. Campos, N.R.C Birkett. An efficient solver for multi-right hand side linear systems based on the CCCG $(\eta)$ method with applications to implicit time-dependent partial differential equations. SIAM J. Sci. Comput, 19:126-138, 1998.

[4] A.R.L. Oliveira and D.C. Sorensen. A new class of preconditioners for large-scale linear systems from interior point methods for linear programming. Linear Algebra and its Applications, 394(0):1 -24, 2005.

[5] M. Velazco, Aurelio R.L. Oliveira, and F.F. Campos. A note on hybrid preconditioners for large-scale normal equations arising from interior-point methods. Optimization Methods \& Software, 25(2):321-332, 2010. 